\title{
Efficient connection strategies in 1D and 2D associative memory models with and without displaced connectivity
}

\author{
Lee Calcraft* , Rod Adams and Neil Davey \\ School of Computer Science, University of Hertfordshire, College lane, Hatfield, Hertfordshire AL10 9AB, United Kingdom
}

\begin{abstract}
This study examines the performance of sparsely-connected associative memory models built using a number of different connection strategies, applied to one- and two-dimensional topologies. Efficient patterns of connectivity are identified which yield high performance at relatively low wiring costs in both topologies. Networks with displaced connectivity are seen to perform particularly well. It is found that two-dimensional models are more tolerant of variations in connection strategy than their one-dimensional counterparts; though networks built with both topologies become less so as their connection density is decreased.
\end{abstract}

Key words: Associative memory models, efficient connection strategies, sparse connectivity, displaced connectivity, 1D and 2D topologies

\section{Introduction}

Our studies of sparsely-connected one-dimensional associative memory models $[1,2,3]$, initially inspired by the work of Watts and Strogatz [4] on the smallworld properties of sparsely-connected systems, demonstrate the importance of the pattern of connectivity between nodes in determining network performance. In a small step towards biological plausibility, we extend our studies to encompass two-dimensional networks. Our associative memory models now represent a 2D substrate of sparselyconnected neurons with a connection density of 0.1 or 0.01 .

We will compare the performance of different connection strategies in our $2 \mathrm{D}$ networks with results obtained from earlier work using a 1D arrangement. This should prove instructive, since 1D treatments of associative memory do not tend to establish to

\footnotetext{
* Corresponding author

Email address: 1.calcraft@herts.ac.uk (Lee Calcraft).
}

Preprint submitted to Elsevier what extent their findings are applicable to more biologically-plausible topologies $[5,6,7,8]$. In this pursuit we acknowledge of course that this study falls short of a full 3D treatment, which would require more processing power than currently available to us.

As with our earlier 1D work, our 2D studies will focus on exploring a variety of connection strategies which achieve good pattern-completion for a minimum wiring length. We are encouraged in this pursuit by recent studies which suggest the importance of wiring optimisation in nature, both from the point of view of the cortical volume taken up by axons and dendrites, the delays and attenuation imposed by long-distance connections, and the metabolic requirements of the connective tissue $[9,10,11]$. A connection strategy which minimises wiring length without impacting upon network performance could potentially mitigate against these unwanted collaterals. It is the goal of the present work to identify such strategies, and to compare their realisations in $1 \mathrm{D}$ and $2 \mathrm{D}$ networks. For this we 
will focus on networks with local, Gaussian and exponential connectivity, and will in addition explore the effect of displacement via axonal-like projection.

\section{Network Dynamics and Training}

Each unit in our networks is a simple, bipolar, threshold device, summing its net input and firing deterministically. The net input, or local field, of a unit, is given by: $h_{i}=\sum_{j \neq i} w_{i j} S_{j}$ where $\mathbf{S}(+/-1)$ is the current state and $w_{i j}$ is the weight on the connection from unit $j$ to unit $i$. The dynamics of the network is given by the standard update:

$$
S_{i}^{\prime}=\left\{\begin{array}{rcc}
1 & \text { if } & h_{i}>0 \\
-1 & \text { if } & h_{i}<0 \\
S_{i} & \text { if } & h_{i}=0
\end{array}\right.
$$

where $S_{i}^{\prime}$ is the new state of $S_{i}$. Unit states may be updated synchronously or asynchronously. Here we use asynchronous, random order updates.

If a training pattern, $\xi^{\mu}$, is one of the fixed points of the network, then it is successfully stored and is said to be a fundamental memory. Given a training set $\left\{\xi^{\mu}\right\}$, the training algorithm is designed to drive the local fields of each unit the correct side of a learning threshold, $T$, for all the training patterns. This is equivalent to requiring that $\forall i, \mu h_{i}^{\mu} \xi_{i}^{\mu} \geq T$.

So the learning rule is given by:

Begin with a zero weight matrix

Repeat until all local fields are correct

Set the state of the network to one of the $\xi^{\mu}$

For each unit, $i$, in turn

Calculate $h_{i}^{\mu} \xi_{i}^{\mu}$

If this is less than $T$ then change the weights on connections into unit $i$ according to:

$$
\forall j \neq i \quad w_{i j}^{\prime}=w_{i j}+C_{i j} \frac{\xi_{i}^{p} \xi_{j}^{p}}{k}
$$

where $\left\{C_{i j}\right\}$ is the connection matrix.

The form of the update is such that changes are only made on the weights that are actually present in the connectivity matrix $\left\{C_{i j}\right\}$ (where $C_{i j}=1$ if $w_{i j}$ is present, and 0 otherwise), and that the learning rate is inversely proportional to the number of connections per unit, $k$. Earlier work has established that a learning threshold $T=10$ gives good results [12], and this is used throughout. Additionally we make no requirement that the connectivity matrix $\left\{C_{i j}\right\}$ should be symmetrical.

\section{Measuring Performance}

The ability to store patterns is not the only functional requirement of an associative memory: fundamental memories should also act as attractors in the state space of the dynamic system resulting from the recurrent connectivity of the network, so that pattern correction can take place.

To measure this we use the Effective Capacity of the network, $E C[8,13]$. The Effective Capacity of a network is a measure of the maximum number of patterns that can be stored in the network with reasonable pattern correction still taking place. We take a fairly arbitrary definition of reasonable as correcting the addition of $60 \%$ noise to within an overlap of $95 \%$ with the original fundamental memory. Varying these figures gives differing values for $E C$ but the values with these settings are robust for comparison purposes. For large fully-connected networks the $E C$ value is proportional to $N$, the total number of nodes in the network, and has a value of approximately 0.1 of the maximum theoretical capacity of the network. For large sparse locally-connected networks, $E C$ is proportional to the number of connections per node, with the constant of proportionality dependent upon the actual connection matrix $C$.

The Effective Capacity of a particular network is determined as follows:

Initialise the number of patterns, $P$, to 0

Repeat

Increment $P$

Create a training set of $P$ random patterns

Train the network

For each pattern in the training set

Degrade the pattern randomly

by adding $60 \%$ noise

With this noisy pattern as the initial state, allow the network to converge Calculate the overlap of the final network state with the original pattern

EndFor

Calculate the mean pattern overlap over all final states

Until the mean pattern overlap is less than $95 \%$

The Effective Capacity is $P-1$. 

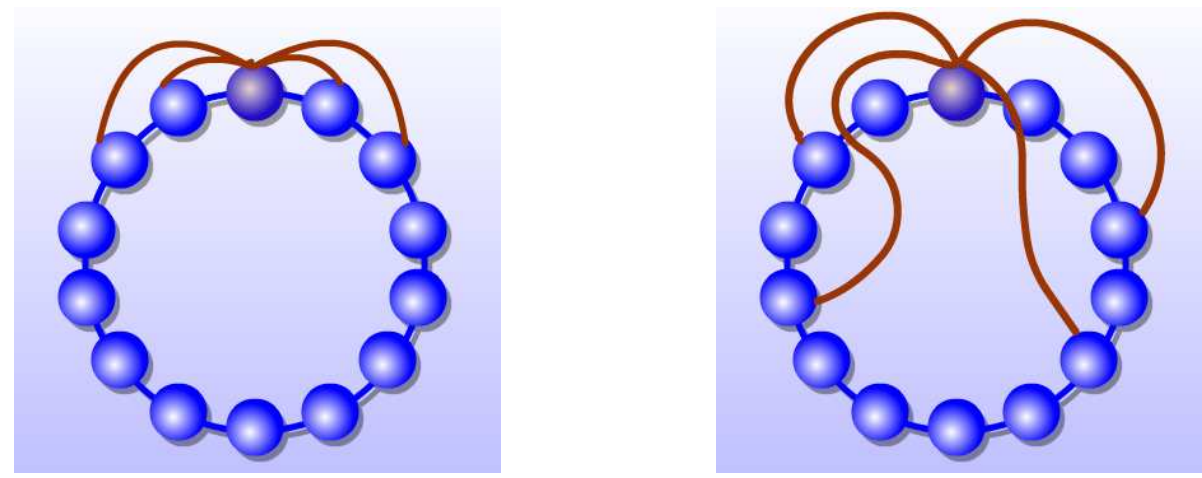

Figure 1. One dimensional sparsely-connected network with 14 nodes, and 4 afferent connections per node, illustrating the connections to a single node: Left, locally-connected, right, after rewiring.
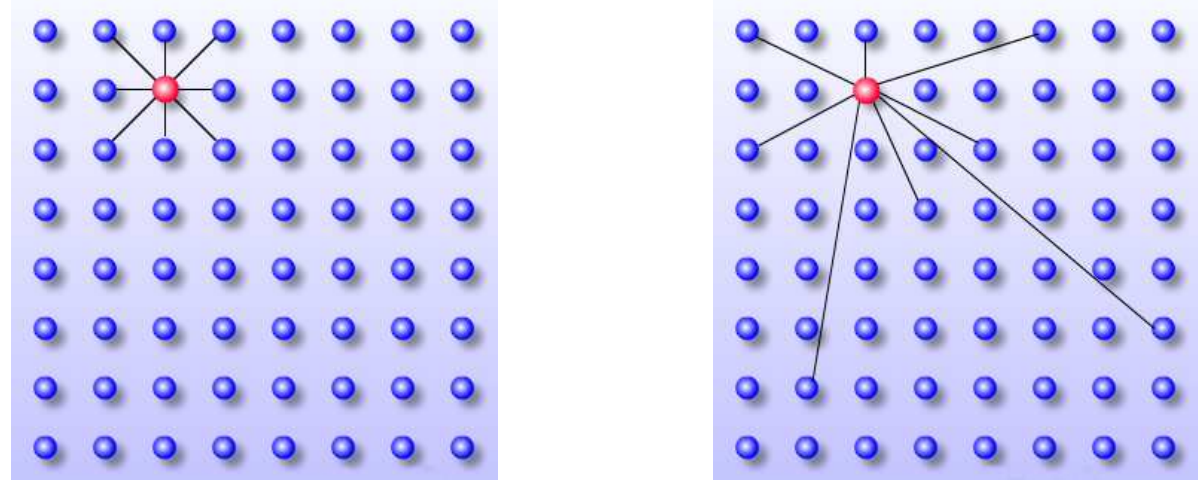

Figure 2. Two dimensional sparsely-connected network with 64 nodes, and 8 afferent connections per node, illustrating the connections to a single node: Left, locally-connected, right, after rewiring. Note that opposite edges are joined to form a toroidal surface.

\section{Network Architecture}

The networks discussed here are based on one- and two-dimensional lattices of $N$ nodes with periodic boundary conditions. Thus the $1 \mathrm{D}$ networks take the form of a ring, and the $2 \mathrm{D}$ implementations that of a torus. The networks are sparse, in which the input of each node is connected to a relatively small, but fixed number, $k$, of other nodes. The main 2D networks examined consist of 4900 nodes arranged in a $70 \times$ 70 array, with 49 afferent (incoming) connections per node, giving a connection density of 0.01 ; and of 484 nodes arranged in a $22 \times 22$ array, with 48 afferent connections per node, giving a connection density of 0.1 . The 1D networks consist of 5000 nodes and of 500 nodes, both with 50 connections per node, again giving connection densities of 0.01 and 0.1 , respectively. All references to spacing refer to the distance between nodes around the ring in the case of the 1D network, or across the surface of the torus in the $2 \mathrm{D}$ case.
We have already established for a 1D network that purely local connectivity results in networks with low wiring length, but with poor patterncompletion performance, while randomly-connected networks perform well, but have high wiring costs [1]. In searching for a compromise between these two extremes we will examine four different connection strategies, applying them to both $1 \mathrm{D}$ and $2 \mathrm{D}$ networks.

\subsection{Progressively rewired}

This is based on the strategy introduced by Watts and Strogatz [4] for generating small-world networks, and applied to a one-dimensional associative memory by Bohland and Minai [7], and subsequently by Davey et al [14]. A locally-connected network is set up, and a fraction of the afferent connections to each node is rewired to other randomly-selected nodes. See figure 1. It is found that rewiring a onedimensional network in this way improves commu- 
nication throughout the network, and that as the degree of rewiring is increased, pattern completion progressively improves, up to the point where about half the connections have been rewired. Beyond this point, further rewiring seems to have little effect [7].

\subsection{Gaussian}

Here the network is constructed in such a way that the probability of a connection between any two nodes separated by a distance $d$ is proportional to

$$
\frac{1}{\sigma} \exp \left(-\frac{(d-1)^{2}}{2 \sigma^{2}}\right)
$$

Network performance is tested for a wide range of values of $\sigma$.

\subsection{Exponential}

In this case the network is set up so that the probability of a connection between any two nodes separated by a distance, $d$, is proportional to

$$
\exp (-\lambda(d-1))
$$

Networks are tested over a wide range of $\lambda$.

\subsection{Displaced connectivity}

Here, inspired by the work of Herzog et al [15], we simulate the effect of an axon which travels some distance from its pre-synaptic neuron before arborisation. Thus in our simulation the connections from any host node are made in a local cluster, separated by a given distance from that node. See figure 3 , which depicts a network of 14 nodes, with 4 connections per node. Figure 3 (left) illustrates local connectivity, while figure 3 (right) shows the effect of displacing the point of arborisation by 3-4 units around the ring. In creating such networks, the direction of displacement is chosen randomly, and we examine the effect on network performance of a range of displacements. A network with zero displacement corresponds to a locally connected network. A similar technique is used with $2 \mathrm{D}$ networks, with displacements of a randomly chosen direction across the 2D surface of the network.

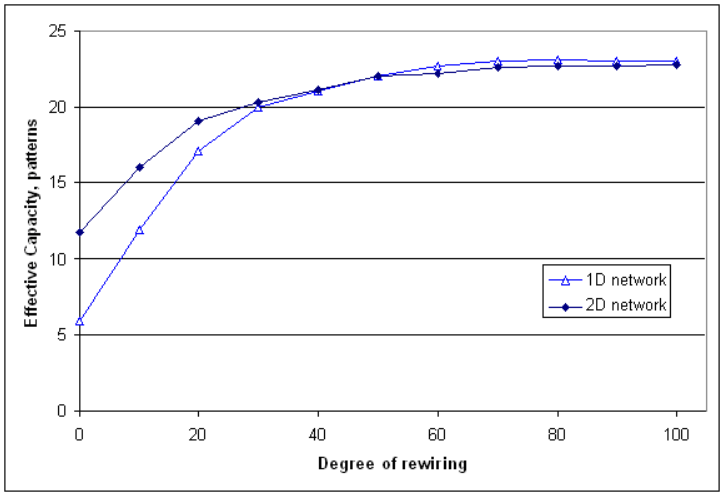

Figure 4. Effective Capacity $v$ s degree of rewiring for a $1 \mathrm{D}$ network with 5000 units and 50 incoming connections per node, and a 2D network with 4900 units and 49 incoming connections per node. The 1D local network has an EC of just 6 , while in the $2 \mathrm{D}$ network it is a much healthier 12 . Once rewiring has reached around 40 or $50 \%$ there is little further improvement in performance.

\section{Results and Discussion}

\subsection{Progressive rewiring}

This connection strategy was introduced by Watts and Strogatz as a way to move in a controlled manner from a locally-connected network to a random one, and as explained above, it involves the progressive rewiring of a locally-connected network to randomly-chosen connection sites. See figure 2 . The results of applying this procedure in $1 \mathrm{D}$ and $2 \mathrm{D}$ networks of similar size are shown in figure 4 . The networks are initially built with local-only connections, and their Effective Capacity is measured as the network is rewired in steps of $10 \%$, until all connections have been rewired, at which point the network is randomly connected. As may be seen, both networks behave similarly, improving in pattern-completion performance as the rewiring is increased, up to around $40 \%$ or $50 \%$ rewiring, after which little further improvement is apparent. This echoes the results reported by Bholand and Minai [7], for a 1D network.

There is, however, an important difference between the performance of the $1 \mathrm{D}$ and $2 \mathrm{D}$ networks here, since although both achieve the same effective Capacity of 23 when fully rewired, their performances are very different when connected locally ( $i e$ when the rewiring is zero). In this configuration the 1D network has an Effective Capacity of 6 patterns, while the 2D network successfully recalls 12 .

In seeking an explanation for this considerable improvement when moving from the $1 \mathrm{D}$ network to the 

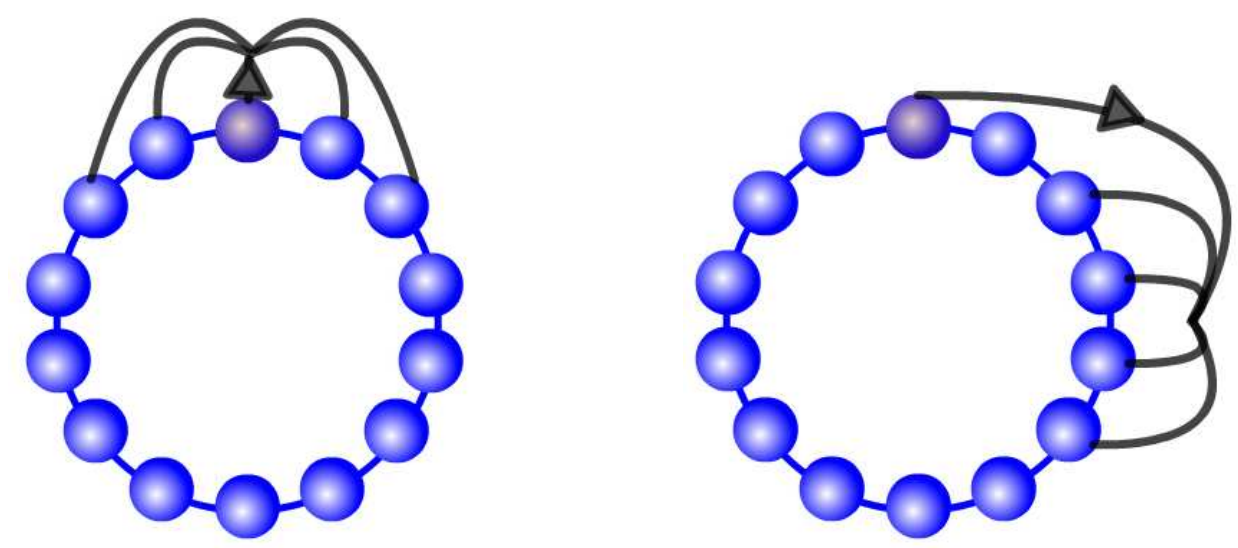

Figure 3. Illustration of a locally-connected network (left), and a network with displaced local connectivity (right). Both networks have 14 nodes, with 4 efferent (outgoing) connections per node. The outgoing connections of only one of the 14 nodes are depicted.

$2 \mathrm{D}$ representation, we would point to two aspects of the network which change as the dimensionality is changed. Firstly, the degree of clustering [4], the extent to which nodes connected to any given node are also connected to each other, decreases from 0.73 to 0.53 as we move from $1 \mathrm{D}$ to $2 \mathrm{D}$ in the above locallyconnected networks; and we have previously found that very tightly clustered networks perform badly as associators [16]. Secondly, there is an improvement in communication across the network as we increase dimensionality. In the 1D network it takes a maximum of 99 steps to pass data between the furthest-separated nodes, whereas in its 2D counterpart this has dramatically dropped to just 9 steps: or translated into terms of characteristic path length [4], the 1D network has a path length of 48 , while in the $2 \mathrm{D}$ network this drops to 6.5 . We would also speculate that in a 3D implementation, a locallyconnected network might perform even better.

The significant improvement in local performance experienced when moving from $1 \mathrm{D}$ to $2 \mathrm{D}$ networks has considerable implications when searching for optimal patterns of connectivity. The reason for this is that, since in the 2D topology there is a much smaller difference between the best and the worst performing architectures, the rewards for using optimal patterns of connectivity will be correspondingly less - and we would speculate that this is likely to be even more significant in $3 \mathrm{D}$ networks.

\subsection{Optimal architectures in networks of connection density 0.01}

In order to compare the performance of other connection strategies with that of progressively-rewired networks, we measured the Effective Capacity of networks whose patterns of connectivity were based on Gaussian and exponential probability distributions of varying $\sigma$ and $\lambda$. The Effective Capacity of all three network types (Gaussian, exponential and progressively-rewired) were then plotted against the mean wiring length of the corresponding networks, providing us with an efficient way to evaluate pattern-completion performance and corresponding wiring costs. Figure 5 shows the results for a $1 \mathrm{D}$ network of 5000 nodes with 50 connections per node, while figure 6 depicts a 2D network of 4900 nodes with 49 connections per node.

We can see from this that in both the 1D and the 2D networks, all three architectures achieve a maximum pattern-completion performance of around 23 patterns. And in both topologies the Gaussian and exponential architectures achieve this at a considerably lower mean wiring length than the progressively-rewired networks. But, largely because of the better performance of the local network in 2D topology, the differences are not so large in the 2D network. Thus, comparing network configurations which achieve an Effective Capacity of 20 (a high value at a relatively low mean wiring length), using a Gaussian architecture in the 1D network would use only one quarter of the wiring of the equivalent progressively-rewired network. In the case of the 2D network, the corresponding saving in 


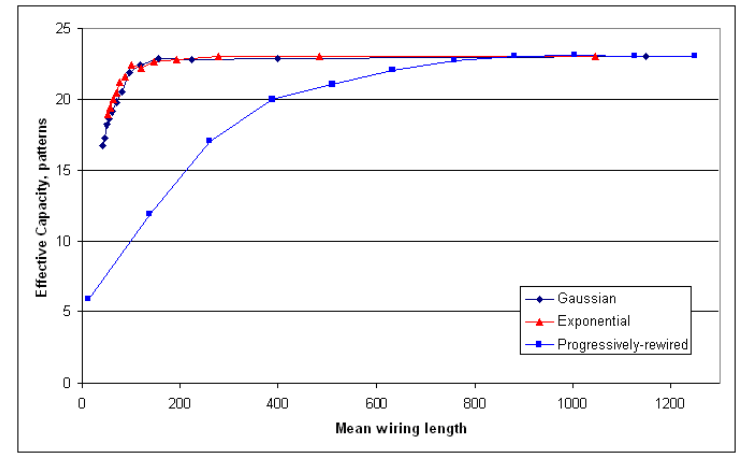

Figure 5. Effective Capacity vs wiring length for Gaussian, exponential and progressively-rewired architectures on a 1D network with 5000 nodes and 50 connections per node. Note that the leftmost point on the rewired plot corresponds to a local-only network (zero rewiring), and the rightmost to a random network (100\% rewiring). Results are averages over 50 runs. The performance of the Gaussian and exponential networks are indistinguishable.

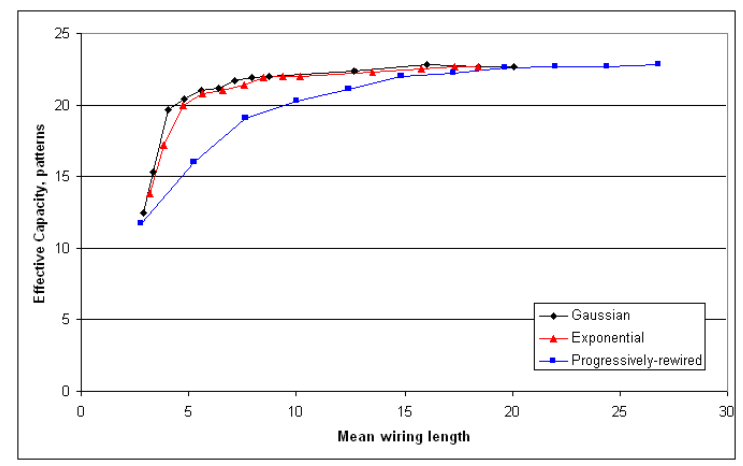

Figure 6. Effective Capacity vs wiring length for Gaussian, exponential and progressively-rewired architectures on a $2 \mathrm{D}$ network with 4900 nodes, and 49 connections per node. Again the leftmost point on the rewired plot corresponds to a local-only network, and the rightmost to a random network. Results are averages over 50 runs.

wiring drops to a half. Clearly, however, this is still far from a trivial saving, and the fact that connectivity between neurons in the cortex is believed to follow a Gaussian probability distribution [17] (ie the probability of any two neurons being connected decreases with distance according to a Gaussian distribution) bears witness to the continuing benefits of this architecture in real 3D systems.

\subsection{Optimal architectures in networks of connection density 0.1}

In our 1D studies using networks of connection density 0.1 we reported that the differences between the rewired network and those based on Gaussian

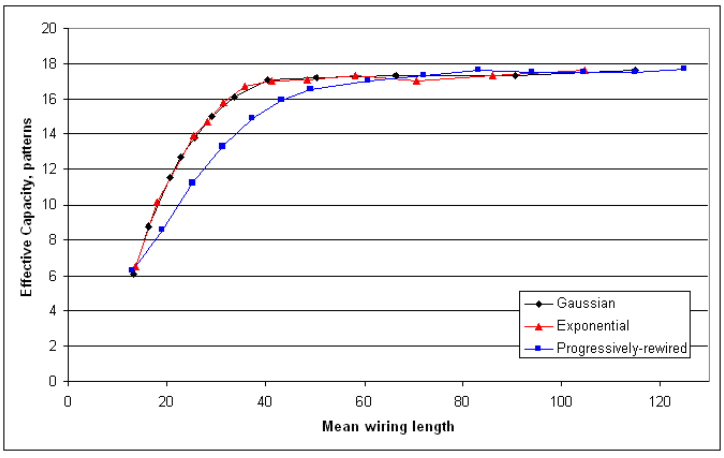

Figure 7. Effective Capacity vs wiring length for Gaussian, exponential and progressively-rewired architectures on a 1D network with 500 nodes, and 50 connections per node. Results are averages over 50 runs.

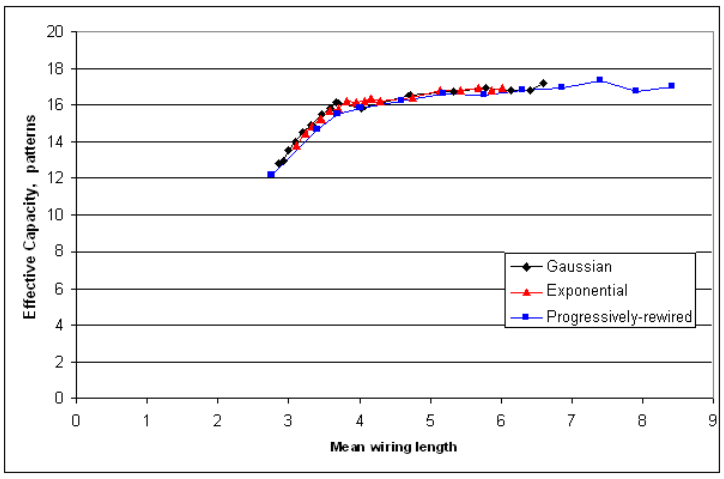

Figure 8. Effective Capacity vs wiring length for Gaussian, exponential and progressively-rewired architectures on a $2 \mathrm{D}$ network with 484 nodes and 48 connections per node. Results are averages over 50 runs.

and exponential distributions were noticeably less than at the lower connection density of 0.01 [1], but that differences were still in evidence. Once we move to a $2 \mathrm{D}$ topology, however, we see that whilst there continues to be a noticeable difference in performance between the rewired network and the Gaussian and exponential distributions at the lower, 0.01 , connection density, this effectively disappears at a connection density of 0.1 . See figure 7 , which illustrates the performance of a 1D network of 500 nodes, with 50 connections per node; and figure 8 , which depicts a $2 \mathrm{D}$ network with 484 nodes, and 48 connections per node.

However, the 2D network on which we are basing this conclusion differs from our previous low connection density 2D network in not one, but two respects. Its connection density is indeed ten times greater, at 0.1 , but the total size of the network is also smaller by a similar factor. Thus it is not yet 


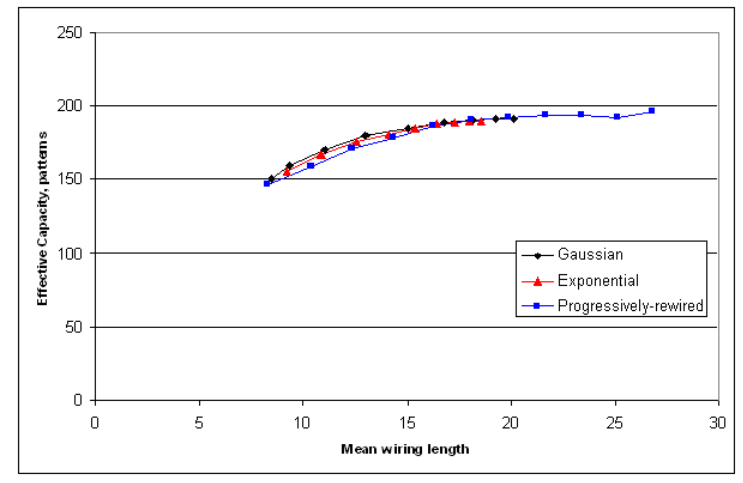

Figure 9. Effective Capacity vs wiring length for Gaussian, exponential and progressively-rewired architectures on a $2 \mathrm{D}$ network with 4900 nodes and 490 connections per node. Results are averages over 50 runs.

clear to what extent the merging of performance of the different architectures seen in the 484 node 2D network is the result of the higher connection density used here (0.1 against 0.01$)$, or whether it is due to the smaller size of the network. In an attempt to distinguish between these two factors, we have repeated the experiment for the $2 \mathrm{D}$ network at a size of 4900 units, with 490 connections per node, thus retaining the higher connection density of 0.1 , but increasing the network size to that used in the lower connection density experiments. The results appear in figure 9 .

Clearly, there is again very little to choose in terms of performance between the three architectures, and we must conclude that in $2 \mathrm{D}$ associative memory models with connection densities of 0.1 and above, whether the pattern of connectivity is based on a Gaussian or exponential probability distribution, or whether a progressively-rewired local network is used, the choice will have very little influence on the pattern-completion performance of the network, or the amount of wiring used.

However, the particular parameters which we adopt (the value of $\sigma$ for a Gaussian distribution, or of $\lambda$ for an exponential, or the degree of rewiring used) will still have considerable influence on performance. These parameters will determine the operating point of our network along the curve in figure 9. At the left-hand end of the curve, a completely local network will give us an Effective Capacity of around 150 patterns, at a mean wiring length of around 8. At the right-hand end we obtain an Effective Capacity of approaching 200 patterns at a mean wiring length of between 20 and 30 .

By contrast, in networks with a connection den- sity of 0.01 , the Gaussian and exponential architectures are clearly better performers than the progressively-rewired network, and because of the relatively steep rise in the Effective Capacity against mean wiring length curves for these architectures, it is easier to select an operation point along the curve which has both a high Effective Capacity and a low mean wiring length.

\subsection{Optimal architectures in networks with displaced connectivity}

We now turn our attention to networks with displaced connectivity. In the foregoing we have assumed that the incoming and outgoing connections to any given node are derived from a simple probability distribution centred on the node. But this is not always the case with neurons in the cortex. It is commonly found that axons travel a certain distance before arborisation [18]. We now factor this behaviour into our simulations, and compare results for both 1D and 2D networks. Figure 3, referred to earlier, illustrates the principle, showing displaced efferent connectivity in a 1D network with 14 nodes.

\section{5. $1 D$ network with displaced connectivity}

For this experiment we use a $1 \mathrm{D}$ network of 500 units, each with 50 efferent connections. We assume that there is initially only a single outgoing connection from each node, which is displaced by a distance laterally before it arborises to connect to the 50 nodes closest to the point of arborisation. The direction of displacement around the ring for any set of connections is random, so that the 'axon' of each node has an equal probability of travelling clockwise or anticlockwise around the ring before arborisation. In the first experiment we measured the Effective Capacity of a locally-connected 1D network with lateral efferent displacements in the range 0 to 200 units. Figure 10 shows the results. The first point on the graph represents the performance of the network with purely local connectivity (displacement is zero). This is poor, as expected. A displacement of 10 units brings a small improvement, but then successive increases reap more considerable rewards, with a reasonably steep linear increase in Effective Capacity as the displacement is increased from 10 to 50 units in steps of 10 . The performance then flattens out at an Effective Capacity of around 16 by the time the displacement has reached 60 or 70 . 


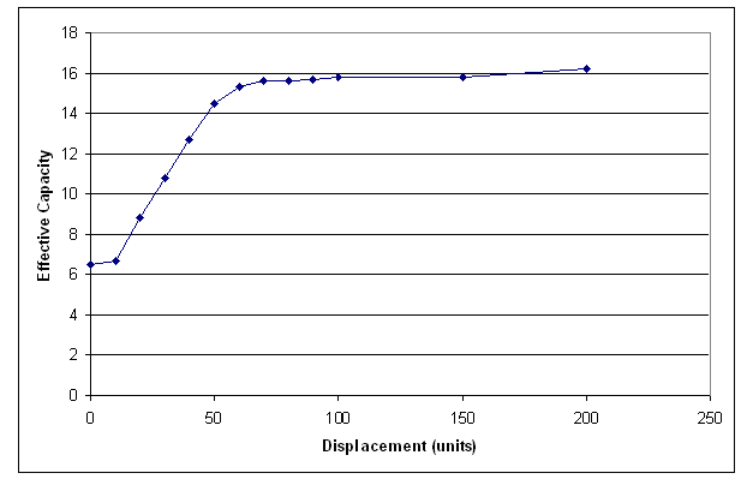

Figure 10. Effective Capacity vs displacement for a locallyconnected network of 500 units, each with 50 efferent connections. Results are averages over 200 runs.

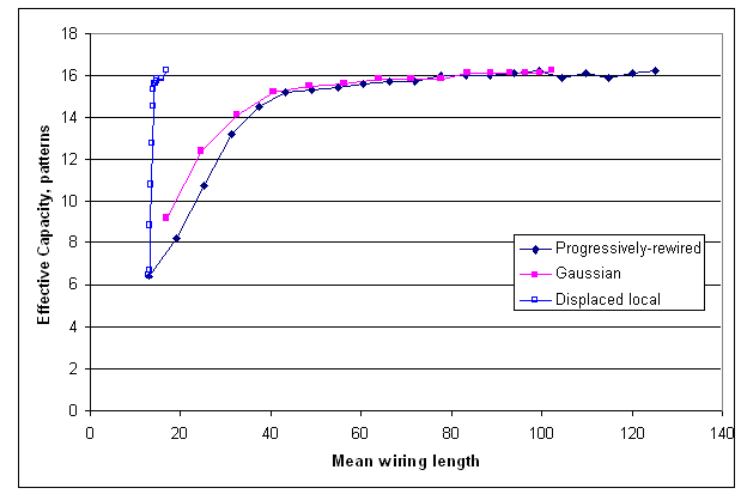

Figure 11. Effective Capacity vs mean wiring length for a 1D network of 500 units, with 50 efferent connections per node. Architectures are based on a local network with increasing displacement from 0 to 200, a progressively-rewired network, and a set of Gaussian distributions of varying width. Results are averaged over 200 runs. The near-vertical slope of the displaced-connectivity plot is caused by the effect of sharing the efferent conduit.

In order to see the effect of displacement of a locally-connected network on performance when wiring costs are taken into account, we again plot Effective Capacity against mean wiring length; and for comparison purposes we have included the Gaussian and progressive rewiring results for an efferent network of the same size. In calculating the wiring length for the displaced network we assume that the output of each node travels along a single connection fibre until it arborises. The results are shown in Figure 11.

As may be seen, the network with displaced connectivity dramatically outperforms the others, reaching an Effective Capacity of more than 16 at a mean wiring length of just 17 units. None of the other networks reach this level of Effective Capac-

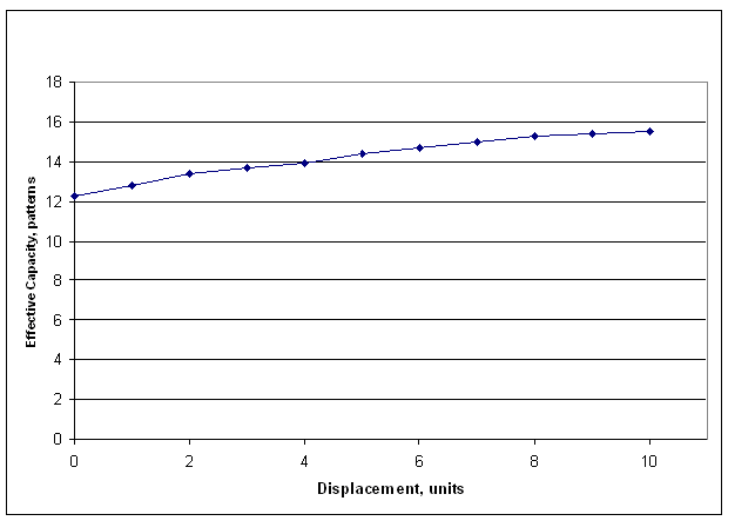

Figure 12. Effective Capacity vs displacement for a 2D network of 484 units, with 48 efferent connections per node. A displacement of 3 units carries an increase in patterns recalled from 12 to nearly 14 .

ity until their mean wiring lengths reach a value of around 80 .

\subsection{D network with displaced connectivity}

In order to see the effect of applying this connection strategy to a more biologically plausible $2 \mathrm{D}$ network, we repeated the experiment with a network arranged as a 2D substrate of $22 \times 22$ nodes (484 nodes in total), each with 48 efferent connections. Figure 12 shows the resultant plot of Effective Capacity against displacement, and demonstrates that, as with the $1 \mathrm{D}$ network, performance improves as the displacement is increased, though to achieve optimum pattern completion performance in this network, the displacement is required to have a value approaching half the width of the substrate. However, improvements are noticeable even for small displacements - thus a displacement of just 3 units takes the Effective Capacity of the network from 12 to nearly 14 .

If we now turn to the overall efficiency of the 2D displaced networks, and plot Effective Capacity against wiring length, we see from figure 13 that the displaced network with efferent connectivity achieves high Effective Capacities for relatively low wiring lengths. As expected, the relative gain in the $2 \mathrm{D}$ network is much smaller than that of its $1 \mathrm{D}$ counterpart ( $c f$ figure 11): the displaced 2D network achieves an Effective Capacity approaching 16 at a mean wiring length of less than 3 , while the Gaussian and rewired networks need nearly double the amount of wiring - which is clearly a non-trivial gain. 


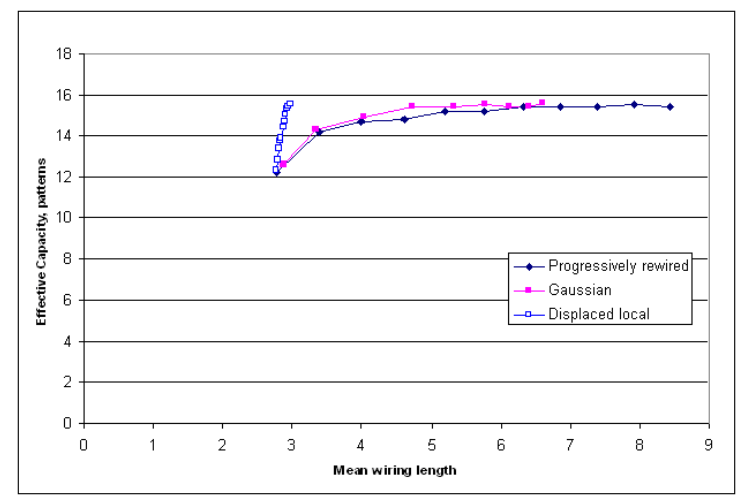

Figure 13. Effective Capacity vs mean wiring length for a 2D network of 484 units, with 48 efferent connections per node. Architectures are based on a local network with increasing displacement from 0 to 10, a progressively-rewired network, and a set of Gaussian distributions of varying width. Results are averaged over 200 runs.

The displaced-local network thus appears to be an interesting contender when attempting to achieve good pattern-completion performance at low wiring costs in both 1D and 2D networks. Moreover, the time which the associator takes to converge during recall in networks with displaced connectivity appears to be comparable to other well-performing networks of similar size (about 8 epochs in the above tests).

The reason for the improved wiring efficiency observed here in networks built with displaced connectivity appears to lie in the sharing of the efferent conduit. In order for an associative memory to perform well, each of its nodes must be connected to some of the nodes which are not immediately local to it [2]. This requirement for non-local connectivity, however, significantly adds to the mean wiring length of the network. But by using displaced arborisation, non-local connectivity can be achieved at a very low wiring cost. These benefits are apparent in networks built with both 1D and 2D topologies, though are greatest in the former.

\section{Conclusion}

Using high capacity associative memory models we have examined the pattern-completion performance and corresponding wiring costs of networks based on a number of different connection strategies, built with a 1D topology. All experiments were repeated for similar networks built with a $2 \mathrm{D}$ topology, and comparisons drawn between the two sets of results.
In our first set of experiments we compared the performance of $1 \mathrm{D}$ and $2 \mathrm{D}$ networks of similar size, as they were progressively rewired from a state of local-only connectivity to a state of fully random connectivity. It was found that although both topologies yielded the same results in the case of random connectivity (as must be the case), there were important differences when connectivity was purely local. In this case the $2 \mathrm{D}$ network was able to recall twice the number of patterns achieved by the $1 \mathrm{D}$ network. It was suggested that this may be the consequence both of the decrease in clustering, and of the much improved communication between distant nodes in the 2D network. It was also suggested that for similar reasons, a 3D network might show even more pronounced effects.

We then compared plots of Effective Capacity against mean wiring length for Gaussian, exponential and progressively-rewired networks. Our initial tests used a connection density of 0.01 . In both the $1 \mathrm{D}$ and $2 \mathrm{D}$ topologies the Gaussian and exponential networks consistently outperformed the progressively-rewired networks, though in moving from a $1 \mathrm{D}$ to a $2 \mathrm{D}$ topology, the benefits of using Gaussian or exponential connectivity were less pronounced.

In networks of connection density 0.1 it was found that the small advantages of using Gaussian or exponential patterns of connectivity over the progressively-rewired network in the $1 \mathrm{D}$ topology all but disappeared in the 2D networks. Thus, while $2 \mathrm{D}$ associative memory models appear to be more tolerant of variations in connection strategy than their 1D counterparts, networks of both types become less so as their connection density is decreased.

Finally we explored the effects of using displaced connectivity, in which the point of efferent arborisation is displaced a finite distance from the host node. Such networks proved to be much more efficient than the other network architectures in achieving a high Effective Capacity at low wiring cost, though once again this strategy proved to be more beneficial in $1 \mathrm{D}$ than in 2D networks. In future work we will investigate the effects of displaced axonal arborisation in networks of even lower connection density. 


\section{References}

[1] L. Calcraft, R. Adams, and N. Davey, Gaussian and exponential architectures in small-world associative memories, Proceedings of ESANN 2006: 14th European Symposium on Artificial Neural Networks. Advances in Computational Intelligence and Learning, pp. 617-622, 2006.

[2] L. Calcraft, R. Adams, and N. Davey, High performance associative memory models with low wiring costs, Proceedings of 3rd IEEE Conference on Intelligent Systems, University of Westminster, 4-6 September 2006-09-29, pp. 612-616, 2006.

[3] L. Calcraft, R. Adams, and N. Davey, Sparselyconnected associative memory models with displaced connectivity, Proceedings of ESANN 2006: 15th European Symposium on Artificial Neural Networks. Advances in Computational Intelligence and Learning, pp. 531-536, 2007.

[4] D. Watts and S. Strogatz, Collective dynamics of smallworld networks, Nature, vol. 393, pp. 440-442, 1998.

[5] P. McGraw and M. Menzinger, Topology and computational performance of attractor neural networks, Physical Review E, vol. 68, pp. 047102, 2003.

[6] F. Emmert-Streib, Influence of the neural network topology on the learning dynamics, Neurocomputing, vol. 69, pp. 1179-1182, 2006.

[7] J. Bohland and A. Minai, Efficient associative memory using small-world architecture, Neurocomputing, vol. 3840, pp. 489-496, 2001.

[8] L. Calcraft, R. Adams, and N. Davey, Locally-connected and small-world associative memories in large networks, Neural Information Processing - Letters and Reviews, vol. 10, pp. 19-26, 2006.

[9] D. Chklovskii, Synaptic connectivity and neuronal morphology: two sides of the same coin, Neuron, vol. 43, pp. 609-617, 2004.

[10] G. Mitchison, Neuronal branching patterns and the economy of cortical wiring, Proceedings: Biological Sciences, vol. 245, pp. 151-158, 1991.

[11] D. Attwell and S. Laughlin, An energy budget for signaling in the grey matter of the brain, Journal of Cerebral Blood Flow and Metabolism, vol. 21, pp. 1133$1145,2001$.

[12] N. Davey, S. P. Hunt, and R. G. Adams, High capacity recurrent associative memories, Neurocomputing, vol. 62, pp. 459-491, 2004.

[13] L. Calcraft, Measuring the performance of associative memories, University of Hertfordshire Technical Report (420) May 2005.

[14] N. Davey, B. Christianson, and R. Adams, High capacity associative memories and small world networks, Proceedings of the IEEE International Joint Conference on Neural Networks, 2004.

[15] A. Herzog, K. Kube, B. Michaelis, A. D. de Lima, and $\mathrm{T}$. Voigt, Connection strategies in neocortical networks, Proceedings of ESANN 2006: 14th European Symposium on Artificial Neural Networks. Advances in Computational Intelligence and Learning, pp. 215-220, 2006.

[16] L. Calcraft, R. Adams, and N. Davey, Efficient architectures for sparsely-connected high capacity associative memory models, Connection Science, vol. 19 , 2007.

[17] B. Hellwig, A quantitative analysis of the local connectivity between pyramidal neurons in layers $2 / 3$ of the rat visual cortex, Biological Cybernetics, vol. 82, pp. 111-121, 2000.

[18] V. Braitenberg and A. Schüz, Cortex: Statistics and Geometry of Neuronal Connectivity. Berlin: SpringerVerlag, 1998. 